\title{
ESGE and ESGENA Position Statement on gastrointestinal endoscopy and COVID-19: An update on guidance during the post- lockdown phase and selected results from a membership survey
}

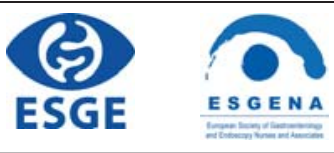

Authors

Ian M. Gralnek ${ }^{1,}{ }^{*}$, Cesare Hassann, ${ }^{*}$, Ulrike Beilenhoff ${ }^{3}$, Giulio Antonelli2, Alanna Ebigbo4 ${ }^{4}$, Maria Pellisé5, Marianna Arvanitakis $^{6}$, Pradeep Bhandari ${ }^{7}$, Raf Bisschops ${ }^{8}$, Jeanin E. Van Hooft ${ }^{9}$, Michal F. Kaminski ${ }^{10}$, Konstantinos Triantafyllou ${ }^{11}$, George Webster ${ }^{12}$, Andrei M. Voiosu ${ }^{13}$, Heiko Pohl' ${ }^{14}$, Irene Dunkley ${ }^{15}$, Björn Fehrke ${ }^{16}$, Mario Gazic ${ }^{17}$, Tatjana Gjergek $^{18}$, Siiri Maasen ${ }^{19}$, Wendy Waagenes ${ }^{20}$, Marjon de Pater ${ }^{21}$, Thierry Ponchon ${ }^{22}$, Peter D. Siersema ${ }^{23}$, Helmut Messmann 4 , Mario Dinis-Ribeiro ${ }^{24}$

Institutions

1 Ellen and Pinchas Mamber Institute of Gastroenterology and Hepatology, Emek Medical Center, Afula, and Rappaport Faculty of Medicine Technion Israel Institute of Technology, Haifa, Israel

2 Nuovo Regina Margherita Hospital, Rome, Italy

3 Ulm, Germany

4 III Medizinische Klinik, Universitätsklinikum Augsburg, Augsburg, Germany

5 Department of Gastroenterology, Hospital Clinic de Barcelona. Institut d'Investigacions Biomediques August Pi i Sunyer (IDIBAPS), and Centro de Investigación Biomédica en Red de Enfermedades Hepáticas y Digestivas (CIBERehd). Universitat de Barcelona, Barcelona, Spain

6 Department of Gastroenterology, Erasme University Hospital, Université Libre de Bruxelles, Brussels, Belgium

7 Gastroenterology, Portsmouth Hospital NHS Trust, Portsmouth, United Kingdom

8 Department of Gastroenterology and Hepatology, Catholic University of Leuven (KUL), TARGID, University Hospitals Leuven, Leuven, Belgium

9 Department of Gastroenterology and Hepatology, Leiden University Medical Center, Leiden, The Netherlands

10 Department of Cancer Prevention and Department of Oncological Gastroenterology, Maria Sklodowska-

Curie National Research Institute of Oncology, Warsaw, Poland

11 Hepatogastroenterology Unit, 2nd Department of Internal Medicine - Propaedeutic, Medical School, National and Kapodistrian University of Athens, Attikon University General Hospital, Athens, Greece
12 Department of Gastroenterology, University College London Hospitals, London, United Kingdom

13 Department of Gastroenterology and Hepatology, Colentina Clinical Hospital, Bucharest, Romania

14 Dartmouth Geisel School of Medicine, Hanover New Hampshire, VA Medical Center, Section of Gastroenterology, White River Junction, Vermont, USA

15 North West Anglia NHS Foundation Trust, Hinchingbrooke, United Kingdom

16 Inselspital, Bern, Switzerland

17 General Hospital, Bjelovar, Croatia

18 University Medical Centre, Ljubljana, Slovenia

19 Tallinn Healthcare College, Tallin, Estonia

20 Hvidovre Hospital, Copenhagen, Denmark

21 Amsterdam UMC location AMC, Amsterdam, The Netherlands

22 Gastroenterology Division, Edouard Herriot Hospital, Lyon, France

23 Department of Gastroenterology and Hepatology, Radboud University Medical Center, Nijmegen, The Netherlands

24 Gastroenterology Department, Portuguese Oncology Institute of Porto, Portugal

published online 8.7 .2020

\# Supplementary material

Online content viewable at:

https://doi.org/10.1055/a-1213-5761

\footnotetext{
* Co-first authors
} 


\author{
Bibliography \\ Endoscopy 2020; 52: 891-898 \\ DOI 10.1055/a-1213-5761 \\ ISSN 0013-726X \\ (C) 2020. Thieme. All rights reserved. \\ Georg Thieme Verlag KG, Rüdigerstraße 14, \\ 70469 Stuttgart, Germany
}

Corresponding author

M. Dinis-Ribeiro, MD, Instituto Português

de Oncologia-Gastroenterologia, Rua Dr. Bernardino de

Almedia, Porto 4200-072, Portugal

mario@med.up.pt

\section{Introduction}

In response to the prolonged lockdown period and implemented measures of social distancing/personal hygiene, most European countries are now (June 2020) experiencing a suppression of the COVID-19 viral pandemic [1]. This post-outbreak sustained (i.e., > 14 days) viral deceleration is being referred to as the "post-lockdown" phase of the COVID-19 pandemic. Sadly, at its height, the COVID-19 pandemic led to innumerable deaths throughout Europe, including many frontline health care professionals (HCPs) fighting the virus [2]. Therefore, we must remain vigilant in protecting HCPs, including our endoscopy unit personnel, by continuing to follow personal protective equipment (PPE) recommendations [3].

It must be stressed however, that the observed deceleration of the viral outbreak is not equivalent to the disappearance of COVID-19 transmission, as a large reservoir of the virus remains present in most European countries [4]. This generates the risk of future COVID-19 outbreaks as the protocols of infection control are gradually relaxed, as multiple viral outbreak clusters in various countries are now showing. Therefore, strategies aiming to triage, separate, and track gastrointestinal (GI) endoscopy patients, as outlined in our original Position Statement, remain highly relevant and necessary in this new post-lockdown phase of the pandemic [3]. An additional new tool, potentially available for $\mathrm{Gl}$ endoscopy units to further assist in patient care, is pre-endoscopy viral testing to more accurately triage patients and/or health care personnel through the identification of COVID-19 viral RNA using nasopharyngeal swabbing [5].

The prolonged lockdown period across Europe resulted in the cancellation of elective $\mathrm{GI}$ endoscopies as only emergent/ urgent endoscopies were performed [6]. As GI endoscopy is largely used for screening, early diagnosis, and treatment of digestive tract cancers, there is a growing concern about a possible mid- or long-term increase in the Gl cancer burden because of the many elective $\mathrm{Gl}$ endoscopy procedures that were cancelled [7]. Competition for endoscopy slots between those cancelled endoscopy procedures and those procedures previously scheduled is now occurring and needs to be addressed. We must do this by striking a careful balance between the ongoing need to maintain infection prevention and control (IPC) protocols within the endoscopy unit and the need to increase endoscopy procedure capacity. Despite there being an excessive backlog of endoscopy cases, there remains no consensus on the best way to efficiently yet safely re-open access for elective endoscopy procedures. Moreover, the status of COVID-19 in
Europe is neither uniform nor generalizable across the continent since some regions of Europe may not yet be in the "post-lockdown" phase of the pandemic.

The aim of this present Position Statement is to provide updated evidence-based guidance on endoscopy practice for the post-lockdown phase of the COVID-19 pandemic. Moreover, in this updated Position Statement, guidance is given on IPC in the post-lockdown period, on the emerging role of COVID-19 viral testing, and on issues related to returning to full endoscopy capacity. Unless otherwise stated, all the guidance statements from the original Position Statement of the European Society for Gastrointestinal Endoscopy (ESGE) and the European Society of Gastroenterology and Endoscopy Nurses and Associates (ESGENA) remain valid and applicable in the post-lockdown phase [3]. Last, to better understand how the COVID-19 pandemic was impacting endoscopy units in Europe, ESGE and ESGENA surveyed their members on the status of their endoscopy practice as related to the COVID-19 pandemic. Thus, in addition to the updated guidance recommendations offered within this Position Statement, we also report selected results from our member survey.

\section{Methods}

As in our original Position Statement, a Pubmed/MEDLINE search was performed once again using 'severe acute respiratory distress syndrome coronavirus 2', 'COVID-19', 'endoscopy, digestive system endoscopy', 'gastrointestinal endoscopic examination, therapy' as MeSH terms between February 1, 2020 and May 31, 2020 to identify relevant publications that could inform this updated Position Statement. When applicable, recommendations by international medical bodies such as the World Health Organization (WHO) and the European and US Centers for Disease Prevention and Control have been considered and adapted as well.

A survey with 35 questions on COVID-19 management in GI endoscopy units was sent to all ESGE individual members in May 2020. The survey was divided into four main content areas (demographics, endoscopy unit organization, personal protective equipment (PPE) use, and training/research). Overall, 595 responses were received, and are summarized in Appendix 1s (available online-only in Supplementary Material). 


\section{A. Infection prevention and control}

\section{STATEMENT}

1 We recommend that during the post-lockdown phase, infection prevention and control (IPC) policies proposed in our original COVID-19 position statement remain in place. These policies apply to IPC training, patient triage, social distancing, isolation, separation, telemedicine, appropriate use of personal protective equipment (PPE), personal hygiene/disinfection, and patient tracking.

\section{STATEMENT}

2 We recommend that IPC interventions should be tailored to the local availability and affordability of resources, while keeping in consideration the local prevalence of COVID-19 and community viral transmission rates.

Endoscopy unit personnel should be considered vulnerable HCPs [8]. This is related to the aerosol-generating nature of GI endoscopy procedures, especially upper endoscopy procedures [9]. Patient triage must be adapted to the new pattern of epidemiology of COVID-19 as defined by WHO ( $\triangleright$ Fig. 1 ) and testing (see below) $[10,11]$. Contact and clustering of the infection, as well as occupational hazards, are now well-defined risk factors for isolation and separation interventions. When and where available, filtering face piece (FFP) respirators (i.e., N95 masks or the equivalent) should be used by the endoscopy unit personnel, regardless of the endoscopic procedure and patient status $[8,12]$.

According to our member survey, however, approximately one third of European Gl endoscopy units reported PPE shortages during the lockdown phase of the pandemic. Where PPE shortages continue to exist, stratification of the risk of viral transmission according to the type of endoscopy procedure is justified, especially in areas with little or no known community transmission of the disease. In addition, most of the responses to our survey consider prolonged upper Gl procedures to have the highest risk for COVID-19 transmission. Patients at high risk for having COVID-19 infection should be isolated and separated within the endoscopy unit to avoid viral exposure of other endoscopy unit patients. However, according to our survey, isolation and separation of patients may be problematic because of the lack of adequate endoscopy unit infrastructure. In such cases, alternative policies, such as separating the morning/ afternoon time slots of patients or alternating the endoscopy unit personnel to minimize their risk of reciprocal viral transmission, are advocated.

Post-endoscopy patient tracking/contact remains of primary importance for IPC because of the relatively long asymptomatic latency period of the COVID-19 virus [13]. Post-endoscopy patient tracking will allow for the potential identification of patients who become symptomatic with COVID-19 following their $\mathrm{Gl}$ endoscopy procedure. According to our member survey, most endoscopy units did not contact or track patients after endoscopy. We believe this should be corrected, and patients should be contacted and tracked following their endoscopy procedure. In some countries, tracking by mobile phone application was to be implemented, representing a possible surrogate.

\section{B. COVID-19 viral testing}

\section{STATEMENT}

1 Given the current lack of high-level evidence, the exclusive use of serology or rapid antigen testing for preendoscopy patient triage cannot be recommended at this time.

\section{STATEMENT}

2 Where readily available, we suggest that pre-endoscopy viral testing based on molecular diagnosis (polymerase chain reaction [PCR] or isothermal nucleic acid amplification [INAAT]) be considered for all patients. See

- Fig. 1.

a) For low prevalence situations and where there are shortages of FFP respirators, it appears reasonable to use a standard surgical mask when performing endoscopy (upper and/or lower Gl endoscopy procedures) in asymptomatic patients thought to be at low risk for having COVID-19 infection combined with a negative PCR test. Use of other PPE (e. g., gloves, hair cover, protective eyewear, waterproof gowns, booties/shoe covers), as recommended in our original Position Statement, should continue to be used [3].

b) Pre-endoscopy viral testing in symptomatic patients, where viral testing is negative, may identify patients so that $\mathrm{Gl}$ endoscopy procedures are not postponed.

\section{STATEMENT}

3 Where there is limited availability of molecular testing, we recommend that viral testing be reserved only for those patients considered to be at high risk for having COVID-19 infection.

\section{Polymerase chain reaction (PCR) testing}

Gastrointestinal endoscopy is an aerosol-generating procedure with a high risk of exposure to pathogens causing acute respiratory infections such as COVID-19 [13]. Stratifying patients according to their probability of having COVID-19 infection based on their self-reported symptoms or risk factors is unreliable. Although viral transmission of COVID-19 usually occurs more commonly from symptomatic patients, transmission of COVID-19 from asymptomatic patients is occurring [14]. Stud- 

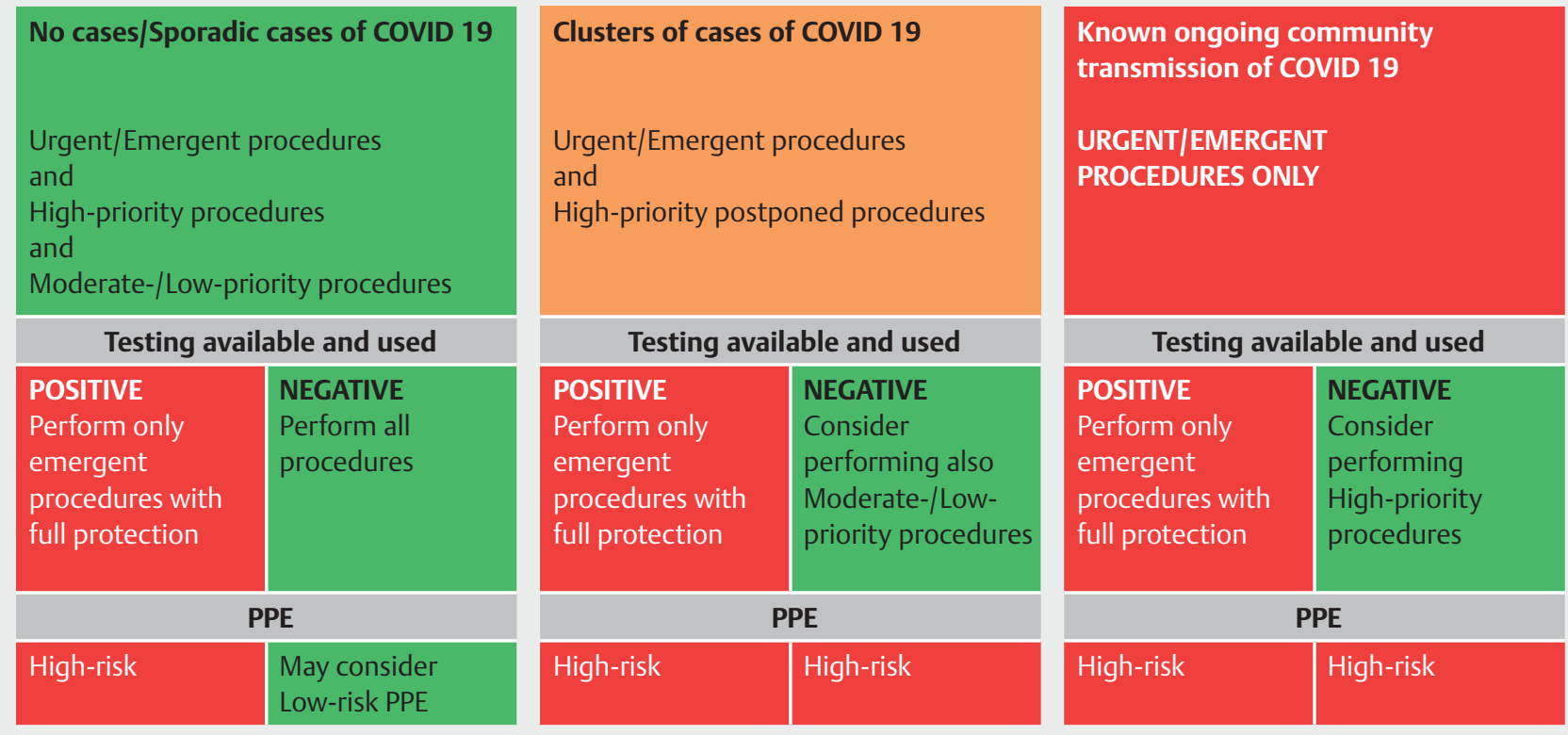

- Fig. 1 Proposal for reopening gastrointestinal endoscopy units according to World Health Organization (WHO) epidemiological regional risk stratification and testing if available [10]. Otherwise follow previous recommendations for use of personal protective equipment (PPE).

ies have shown varying rates of asymptomatic COVID-19-infected persons [16-18]. For example, in a population screening study from Iceland, $43 \%$ of COVID-19-positive persons were asymptomatic at the time of diagnosis [19]. The relatively long incubation period, that may last up to 2 weeks, and the suboptimal accuracy of the clinical signs/symptoms for the diagnosis of COVID-19, may explain the high rate of asymptomatic or possibly presymptomatic persons [20].

Molecular diagnosis based on direct identification of viral RNA with reverse transcription (RT)-PCR or with isothermal nucleic acid amplification testing (INAAT) has demonstrated, at least in vitro, high levels of sensitivity and specificity of $\geq 95 \%$ [21-24]. Additional advantages of molecular viral diagnosis are the potential to create efficient point-of-care testing and the short time for processing test results. Coupling the high accuracy with the relatively low prevalence of the disease, the negative predictive value approaches $100 \%$.

A meta-analysis of the effectiveness of face masks has suggested that the use of FFP-2/N95 respirators compared with the use of standard surgical masks is not associated with a statistically significant lower risk of viral infection, at least in nonaerosol-generating procedures [25]. In our survey, nearly one third of respondents favored the integration of pre-endoscopy viral testing. However, a pre-endoscopy testing policy might not be easily implemented in endoscopy units because of multiple barriers. First, there is the need for an adequate organizational structure as patients after being tested, must remain self-isolated for 48-72 hours to prevent undetectable preendoscopy infection. Second, most countries suffer from shortages of testing reagents or manpower when considering the high volume of testing that is performed at the population level to identify infected patients. Third, most endoscopy cen- ters do not have the availability and/or the affordability of inhouse PCR. Fourth, endoscopy centers must have a policy for dealing with false-positive test results that could be higher than the true-positive results [26]. Finally, it must be taken into consideration that viral testing can result in a false-negative test, especially when the nasopharyngeal swab is not performed correctly or in patients with a low pharyngeal viral load. For situations in which pre-endoscopy viral testing is not routinely or readily available, viral testing should be reserved for those patients thought to be at high risk of having COVID19 infection based on their pre-endoscopic IPC risk stratification as previously recommended by ESGE-ESGENA [3].

\section{Antigen and serology testing}

Despite its biological plausibility and preliminary in vitro results, the initial clinical experience of immunological identification of the COVID-19 antigen from nasopharyngeal swabbing has shown suboptimal results in terms of sensitivity as compared with PCR [27]. If any of the antigen detection tests that are currently under development or subsequently commercialized demonstrate adequate performance characteristics, they could potentially be used as pre-endoscopy triage tests to rapidly identify patients who are very likely to have COVID-19, thereby reducing or eliminating the need for expensive molecular confirmatory testing [28]. Despite the availability of serology testing in most countries, its use to risk-stratify the pre-endoscopy patient is marginal because of the window period before infected individuals develop antibodies [29].

Testing can be considered for endoscopy unit personnel when there has been a breach of IPC, such as when an assumed COVID-19-negative patient becomes positive during postendoscopy tracking or where there has been incorrect use of 
PPE with COVID-19-positive patients, or any time an HCP develops COVID-19-like symptoms.

\section{Restarting GI endoscopy}

\section{STATEMENT}

1 We recommend that during the post-lockdown phase, a return to full $\mathrm{Gl}$ endoscopy procedure capacity be pursued in those areas without evidence of community transmission of COVID-19, while continuing to adhere to IPC measures.

\section{STATEMENT}

2 We recommend that prioritization of Gl endoscopy procedures by clinical or oncological indication should be required in those areas with limited endoscopic capacity.

\section{STATEMENT}

3 We recommend that patients' fears of contracting COVID-19 infection while visiting a GI endoscopy unit should be properly addressed. This especially includes having in place appropriate protective policies for those patients at high risk of contracting COVID-19 infection or of having poor outcomes from COVID-19 infection (e.g., elderly, having comorbidities, immunocompromised).

\section{STATEMENT}

4 We recommend that in the post-lockdown phase, GI endoscopy units involved in endoscopy training and research activities gradually restart their endoscopy training programs and research activities, provided these activities will not further delay needed GI endoscopic procedures.

According to our member survey (Appendix 1s), most of the endoscopy units reported a substantial decrease in the volume of $\mathrm{Gl}$ endoscopy procedures performed during the lockdown phase ( $75 \%$ to $100 \%$ endoscopy volume reduction). This decrease is explained by the fact that most endoscopy units limited their endoscopic activity to urgent indications only.

In addition, most endoscopy units are expecting insufficient capacity/manpower/local resources when endoscopy activity is restarted. This may be explained by at least two factors: (i) there is an overlap between the rescheduled lockdown endoscopy patient list and the new endoscopy appointments to be scheduled, generating a competition between the endoscopy waiting lists; (ii) IPC policies prevent a full return to endoscopy capacity because of the longer times required for room disinfection and the ongoing separation of intake/outbound pathways within the endoscopy unit for COVID-19 patients [5, 8]. There is concern however, about missed/preventable diagnosis of Gl cancers if endoscopy procedures are inordinately delayed [7].

For this reason, priority stratification of Gl endoscopy procedures according to indication is recommended ( $>$ Table $\mathbf{1}$ ). Priority for endoscopy should be given to those patients with alarm symptoms or those at higher risk of $\mathrm{Gl}$ cancer based upon previous examination findings/results (e.g. positive fecal immunochemical test [FIT], radiographic suspicion or evidence). Conversely, surveillance of patients at average or reduced risk of cancer, such as post-polypectomy or post-surgery colorectal cancer (CRC) patients may continue to be temporarily postponed. In addition, the opening of new time-slots for rescheduled endoscopy procedures must be considered based upon local availability and resources.

An additional factor to be considered is patients' fear of being infected with the COVID-19 virus while visiting the GI endoscopy unit. For this reason, policies aimed at protecting patients at higher risk of contracting COVID-19 and having poor outcomes from COVID-19 infection (e.g., elderly, having comorbidities, immunocompromised) are recommended and acceptable. Alternatively, direct patient contact through telemedicine should encourage patients at high risk for GI disease/ malignancy, such as those with a positive FIT, to undergo endoscopy. For patients extremely reluctant to undergo endoscopy, non-endoscopic testing may be considered.

According to our survey, the great majority of endoscopy training programs were interrupted during the COVID-19 pandemic lockdown period. Many respondents reported that a general reduction in endoscopic skill acquisition was expected, affecting every domain of endoscopic activities. For trainees in endoscopy, supplementation of hands-on training with educational alternatives (e.g. endoscopy simulator or animal models where available) could mitigate the potentially detrimental effect on skill acquisition due to the decreased volume of endoscopy procedures involving trainees. We must remember that gastrointestinal endoscopy is not simply a technical discipline, it is also cognitive. Thus, trainees can also further develop their cognitive endoscopic skills by use of online learning and viewing of endoscopy videos and technical training videos [7]. In addition, according to the ESGE survey, research activities have been delayed or completely suspended, apart from specific COVID-19 endoscopy-related projects. For these reasons, a return to normal endoscopy training schedules and research activities is desirable, provided no further delays in Gl endoscopy procedure rescheduling are caused. 
- Table 1 Prioritization of gastrointestinal (GI) endoscopy procedures stratified by clinical indication.

Urgent/emergent

High priority
Acute upper/lower GI bleeding with hemodynamic instability

Capsule/enteroscopy for urgent/emergent bleeding

Anemia with hemodynamic instability

Foreign body in esophagus and/or high-risk foreign body in the stomach

Obstructive jaundice

Acute ascending cholangitis

Endoscopic treatment of high-grade dysplasia (HGD) or early intramucosal cancer in the esophagus, stomach, or large colonic polyps at high risk of submucosal invasion

Malignant stricture stenting

Percutaneous endoscopic gastrostomy (PEG)/Percutaneous endoscopic jejunostomy (PEJ)/Nasojejunal tube Upper GI fistula/leakage

Dysphagia or dyspepsia with alarm symptoms present

Upper GI bleeding without hemodynamic instability

Rectal bleeding

Colonoscopy for melena after negative upper Gl endoscopy

Severe anemia with no hemodynamic instability

Tissue acquisition needed for the initiation of systemic therapy/surgery

Colonoscopy within organized positive fecal occult blood test (FOBT)/fecal immunochemical test (FIT) colorectal cancer (CRC) screening program

Foreign body in the stomach, low-risk

Benign stricture requiring dilation/stenting

Radiologic evidence of mass

Lymph node endoscopic ultrasound (EUS) sampling

Gallstone-related pancreatitis

Pancreatic mass/stricture

Biliary stricture dilation

Pancreaticobiliary stent replacement

Necrosectomy

Endoscopic treatment of esophageal or gastric low-grade dysplasia (LGD)

Duodenal polyp

Ampullectomy

Band ligation/non-emergency

Iron-deficiency anemia

Pancreatic cyst (depending on risk features)

Biliary stricture/no urgency (no cholangitis, no jaundice, etc.)

Submucosal lesion EUS sampling

Achalasia (peroral endoscopic myotomy [POEM], balloon dilation)

Positive guaiac fecal occult blood test (gFOBT)/FIT, outside of an organized regional/ national screening program 

sclerosing cholangitis

Post endoscopic or surgical resection of cancer

Post-polypectomy surveillance

Diagnosis/Surveillance of hereditary nonpolyposis colorectal cancer (HNPCC) and other hereditary syndromes

Workup of irritable bowel syndrome (IBS)-related symptoms

Workup of reflux disease, dyspepsia (no alarm symptoms)

Screening in high-risk patients for esophageal, stomach or colon cancer

Bariatric endoscopic surgery

\section{Disclaimer}

ESGE position statements represent a consensus of best practice based on the available evidence at the time of preparation. They may not apply in all situations and should be interpreted in the light of specific clinical situations and resource availability. Further controlled clinical studies may be needed to clarify aspects of these statements, and revision may be necessary as new data appear. Clinical considerations may justify a course of action at variance to these statements. ESGE position statements are intended to be an educational device to provide information that may assist endoscopists in providing care to patients. They are not rules and should not be construed as establishing a legal standard of care or as encouraging, advocating, requiring, or discouraging any particular treatment.

\section{Competing interests}

I.M. Gralnek has received lecture fees from AstraZeneca, Taro Pharma, Vifor Pharma, and 3D Matrix (ongoing), and consultancy fees from Boston Scientific, GI View, Motus GI, and Symbionix (ongoing); he is a member of the Medical Advisory Board of Motus GI. J.E. van Hooft has received lecture fees from Medtronics (2014-2015) and Cook Medical (2019), and consultancy fees from Boston Scientific (2014-2017); her department has received research grants from Cook Medical (2014-2019) and Abbott (2014-2017). M. Kaminski has received lecture fees from Boston Scientific (2018- ongoing) and consultancy fees from Olympus and Fujifilm (2018- ongoing). P. D. Siersema has received research grants from Norgine, Pentax, Microtech, Yakult, and Motus Gl (ongoing); he is a member of the Medical Advisory Board of Motus Gl. G. Antonelli, M. Arvanitakis, P. Bhandari, R. Bisschops, M. Dinis-Ribeiro, A. Ebigbo, C. Hassan, H. Messmann, M. Pellisé, H. Pohl, T. Ponchon, K. Triantafyllou, A. M. Voiosu, and $\mathrm{G}$. Webster, have no competing interests.

\section{References}

[1] European Centre for Disease Prevention and Control. COVID-19 situation update worldwide, as of 3 June 2020. https://www.ecdc. europa.eu/en/geographical-distribution-2019-ncov-cases Accessed: 3 Jun 2020

[2] In memoriam: Healthcare workers who have died of COVID-19. Medscape. https://www.medscape.com/viewarticle/927976 Accessed 1 May 2020.
[3] Gralnek IM, Hassan C, Beilenhoff U et al. ESGE and ESGENA Position Statement on gastrointestinal endoscopy and the COVID-19 pandemic. Endoscopy 2020; 52: 483-490

[4] European Centre for Disease Prevention and Control. COVID-19 situation update for the EU/EEA and the UK, as of 3 June 2020. https:// www.ecdc.europa.eu/en/cases-2019-ncov-eueea Accessed: 3 Jun 2020.

[5] Gupta S, Shahidi N, Gilroy N et al. Proposal for the return to routine endoscopy during the COVID-19 pandemic. Gastrointest Endosc 2020: doi:10.1016/j.gie.2020.04.050

[6] Repici A, Pace F, Gabbiadini R et al. Endoscopy units and the COVID-19 outbreak: a multi-center experience from Italy. Gastroenterology 2020: doi:10.1053/j.gastro.2020.04.003

[7] Gralnek IM, Hassan C, Dinis-Ribeiro M. COVID-19 and endoscopy: implications for healthcare and digestive cancer screening. Nat Rev Gastroenterol Hepatol 2020: doi:10.1038/s41575-020-0312-x

[8] Repici A, Maselli R, Colombo M et al. Coronavirus (COVID-19) outbreak: what the department of endoscopy should know. Gastrointest Endosc 2020; 92: 192-197 doi:10.1016/j.gie.2020.03.019

[9] Lui RN, Wong SH, Sánchez-Luna SA et al. Overview of guidance for endoscopy during the coronavirus disease 2019 pandemic. J Gastroenterol Hepatol 2020; 35: 749-759

[10] World Health Organization. Critical preparedness, readiness and response actions for COVID-19. https://www.who.int/publications/i/ item/critical-preparedness-readiness-and-response-actions-for-covid-19

[11] World Health Organization. Considerations in adjusting public health and social measures in the context of COVID-19: interim guidance. https://www.who.int/publications-detail/considerations-in-adjusting-public-health-and-social-measures-in-the-context-of-covid-19interim-guidance

[12] Sultan S, Lim JK, Altayar O et al. AGA Institute rapid recommendations for gastrointestinal procedures during the COVID-19 pandemic. Gastroenterology 2020: doi:10.1053/j.gastro.2020.03.072

[13] World Health Organization. Contact tracing in the context of COVID19. https://www.who.int/publications-detail/contact-tracing-in-thecontext-of-covid-19

[14] Tran K, Cimon K, Severn M et al. Aerosol generating procedures and risk of transmission of acute respiratory infections to healthcare workers: a systematic review. PLoS One 2012; 7: e35797 doi:10.1371/ journal.pone.0035797

[15] Wang Y, Tong J, Qin Y et al. Characterization of an asymptomatic cohort of SARS-COV-2 infected individuals outside of Wuhan, China. Clin Infect Dis 2020: doi:10.1093/cid/ciaa629

[16] Epidemiology Working Group for NCIP Epidemic Response, Chinese Center for Disease Control and Prevention. [The epidemiological characteristics of an outbreak of 2019 novel coronavirus diseases 
(COVID-19) in China]. Zhonghua Liu Xing Bing Xue Za Zhi 2020; 41: 145-151 doi:10.3760/cma.j.issn.0254-6450.2020.02.003

[17] Mizumoto K, Kagaya K, Zarebski A et al. Estimating the asymptomatic proportion of coronavirus disease 2019 (COVID-19) cases on board the Diamond Princess cruise ship, Yokohama, Japan, 2020. Euro Surveill 2020; 25: doi:10.2807/1560-7917.ES.2020.25.10.2000180

[18] Nishiura H, Kobayashi T, Miyama T et al. Estimation of the asymptomatic ratio of novel coronavirus infections (COVID-19). Int J Infect Dis 2020; 94: 154-155

[19] Gudbjartsson DF, Helgason A, Jonsson H et al. Spread of SARS-CoV-2 in the Icelandic population. N Engl J Med 2020; 382: 2302-2315

[20] Gao Z, Xu Y, Sun C et al. A systematic review of asymptomatic infections with COVID-19. J Microbiol Immunol Infect 2020: May 15; S1684118220301134. doi:10.1016/j.jmii.2020.05.001

[21] Wölfel R, Corman VM, Guggemos W et al. Virological assessment of hospitalized patients with COVID-2019. Nature 2020; 581: 465-469

[22] Smithgall MC, Scherberkova I, Whittier S et al. Comparison of Cepheid Xpert Xpress and Abbott ID Now to Roche Cobas for the rapid detection of SARS-CoV-2. J Clin Virol 2020; 128: 104428 doi:10.1016/j. jcv.2020.104428

[23] Corman VM, Landt O, Kaiser M et al. Detection of 2019 novel coronavirus (2019-nCoV) by real-time RT-PCR. Euro Surveill 2020; 25 : 2000045 doi:10.2807/1560-7917.ES.2020.25.3.2000045
[24] Pfefferle S, Reucher S, Nörz D et al. Evaluation of a quantitative RTPCR assay for the detection of the emerging coronavirus SARS-CoV-2 using a high throughput system. Euro Surveill 2020; 25: 2000152 doi:10.2807/1560-7917.ES.2020.25.9.2000152

[25] Long Y, Hu T, Liu L et al. Effectiveness of $\mathrm{N} 95$ respirators versus surgical masks against influenza: A systematic review and meta-analysis. J Evid Based Med 2020; 13: 93-101

[26] Corral JE, Hoogenboom SA, Kröner PT et al. COVID-19 polymerase chain reaction testing before endoscopy: an economic analysis. Gastrointest Endosc 2020: S0016-5107(20)34248-6. doi:10.1016/j. gie.2020.04.049

[27] Mertens P, De Vos N, Martiny D et al. Development and potential usefulness of the COVID-19 Ag Respi-Strip diagnostic assay in a pandemic context. Front Med (Lausanne) 2020: doi:10.3389/ fmed.2020.00225

[28] World Health Organization. Advice on the use of point-of-care immunodiagnostic tests for COVID-19: scientific brief. https://www. who.int/publications/i/item/advice-on-the-use-of-point-of-care-immunodiagnostic-tests-for-covid-19-scientific-brief

[29] Krammer F, Simon V. Serology assays to manage COVID-19. Science 2020; 368: 1060-1061 doi:10.1126/science.abc1227 